Die früheste Phase im Leben eines Menschen ist für seine gesundheitliche Entwicklung von entscheidender Bedeutung. Es ist aus Public-Health-Perspektive dringend, gesundheits- und entwicklungsförderliche Rahmenbedingungen zur Verfügung zu stellen, um Kinder in ihren ersten Lebensjahren im Sinne des Resilienzkonzeptes für die Bewältigung künftiger Herausforderungen zu stärken. Junge Familien verdienen es, bei der Bewältigung der anspruchsvollen Aufgabe unterstützt zu werden, die Kinder auf die Schule und ihr weiteres Leben vorzubereiten. Mit ihrem Positionspapier formuliert Public Health Schweiz sieben gesundheitspolitische Forderungen für einen Ausbau der frühen Förderung und begründet diese anhand aktueller wissenschaftlicher Übersichtsarbeiten.

\title{
Strategische Ziele reichen nicht, Handeln ist angesagt
}

\author{
Oskar Jenni \\ Prof. Dr. med., Leiter der Abteilung Entwicklungspädiatrie, Kinderspital Zürich
}

Die Lebensbedingungen haben sich für die Kinder in der Schweiz in den letzten Jahrzehnten stetig verbessert. Wir haben im Vergleich zu anderen Ländern ein hochstehendes Gesundheits- und Bildungssystem, eine niedrige Arbeitslosigkeit und eine relativ geringe Armutsquote. Das sind entscheidende entwicklungsund gesundheitsfördernde Rahmenbedingungen für Kinder und Jugendliche. Aber auch in der Schweiz leben Kinder in schwierigen psychosozialen Verhältnissen oder sie leiden an Entwicklungs- und Verhaltensauffälligkeiten.

\section{Welche Kinder sind betroffen?}

Schätzungsweise fünf Prozent der Kinder sind schwerwiegend von einer Entwicklungsstörung betroffen, beispielsweise Kinder mit geistiger Behinderung, Autismus, Bewegungsstörung oder Sprachbehinderung. Diese Kinder brauchen Therapien oder Fördermassnahmen. Es gibt daneben aber viele Kinder mit Besonderheiten, die nur schwer von der normalen Entwicklung abgegrenzt und eher als spezielle Ausprägung oder Variante der Norm bezeichnet werden können. Diese Gruppe von Kindern ist in den letzten Jahren grösser geworden, weil die Sensibilität für leichtere Entwicklungsauffälligkeiten gestiegen ist und das Therapieangebot ausgeweitet wurde.

\section{Welche Massnahmen sind nötig?}

Im Vordergrund stehen weniger spezifische Unterstützungsmassnahmen oder Therapien, sondern vielmehr die Förderung elterlicher Kompetenz in Gesundheits-, Entwicklungs- und Erziehungsfragen. Das ist auch eine der Forderungen des Positionspapiers "Gesundheitsförderung in der frühen Kindheit». Eltern sind in der heutigen Zeit sehr belastet, weil der gesellschaftliche Anspruch lautet, dass sie für eine perfekt gelingende Erziehung und Entwicklung ihrer Kinder verantwortlich sind. Nicht alle Eltern sind diesen Anforderungen allerdings gewachsen. Die Begleitung von Familien und die Elternbildung sind zentrale frühkindliche Präventionsaufgaben.

\section{Forschungsschwerpunkte zur Gesund- heitsförderung im frühen Kindesalter}

Zum einen braucht es vermehrt Untersuchungen zu den Mechanismen von Risiko- und Schutzfaktoren für eine gesunde Entwicklung im frühen Kindesalter. In der vom Schweizerischen Nationalfonds und der Jacobs Foundation unterstützten Swiss Preschoolers Health Study (SPLASHY) untersuchen wir zusammen mit den Universitäten Zürich, Lausanne und Fribourg in einer grossen nationalen Kohorte den Einfluss von Stress und Bewegung auf die psychische Gesundheit und die körperliche und geistige Entwicklung von 3-5-jährigen Kindern, die Kindertagesstätten besuchen. Ferner sind Studien zur Wirksamkeit von gesundheits- und entwicklungsfördernden Massnahmen notwendig. So wird zum Beispiel im Projekt ZEPPELIN der Zürcher Hochschule für Heilpädagogik die Wirksamkeit früher Förderung bei Familien in

\section{Zum Autor}

Der Kinderarzt Oskar Jenni leitet seit 2005 die Abteilung Entwicklungspädiatrie des Kinderspitals Zürich. Er studierte an den Universitäten Zürich und Stellenbosch (Südafrika) Medizin und arbeitete als Entwicklungspädiater an der amerikanischen Brown University in Providence, Rhode Island. Er ist Titularprofessor für Entwicklungspädiatrie an der Universität Zürich und Vorstandsmitglied der Schweizerischen Gesellschaft für Pädiatrie. 
psychosozialen Risikosituationen untersucht. Solche Studien sind unerlässlich, damit die politisch Verantwortlichen überzeugt werden können, entsprechende Programme in die Regelversorgung aufzunehmen und zu finanzieren.

\section{Gute Vernetzung ist wichtig}

Eine bestmögliche Versorgung der Familien kann nicht nur durch eine Fachperson geleistet werden, so engagiert und kompetent sie auch sein mag. Eine gute Vernetzung der verschiedenen Angebote ist notwendig. Im Frühbereich sind die Kindermedizin, die heilpädagogische Früherziehung, die Logopädie, sozialpädagogische und psychologische Dienste sowie die Mütter- und Väterberatung die zentralen Versorgungspartner. Besonders wichtig erscheint das Überbrücken der Grenzen zwischen diesen Disziplinen, was sich im Alltag aber immer wieder als grosse Herausforderung herausstellt.

\section{Wichtige Themenfelder}

Das Positionspapier sieht den Bereich Elternbildung, die Themenfelder Beziehungsaufbau, Spracherwerb, Ernährung, Bewegung sowie die Gestaltung einer anregenden und lernfördernden Umgebung vor. Das sind die wichtigsten Themen in der frühen Kindheit, und sie entsprechen auch den Bedürfnissen kleiner Kinder.

\section{Die Rolle der Grundversorger}

Ärztinnen und Ärzte geniessen als Experten für die körperliche und psychische Gesundheit und Entwicklung von Kindern in der Regel ein hohes Vertrauen und Glaubwürdigkeit bei Eltern und Erziehungsberechtigten. Sie spielen deshalb in der Elternbildung eine zentrale Rolle. Sie kennen die Familien meist seit Geburt und sehen die Kinder im Rahmen von Impfungen regelmässig in den Vorsorgeuntersuchungen. Der Elternberatung sollte bei diesen regelmässigen Besuchen mindestens ebenso viel Beachtung geschenkt werden wie dem Entwicklungsscreening. Es gibt in der Zwischenzeit empirische Studien, welche die Wirksamkeit und Wirtschaftlichkeit der Elternberatung belegen. So konnte beispielsweise in einer amerikanischen Untersuchung gezeigt
Positionspapier «Gesundheitsförderung im frühen Kindesalter»

Ausgearbeitet durch die Fachgruppe Gesundheitsförderung von Public Health Schweiz, am 22. November 2012 vom Zentralvorstand genehmigt. Kontakt: Prof. Felix Wettstein, Fachhochschule Nordwestschweiz, Hochschule für Soziale Arbeit, Riggenbachstrasse 16, 4600 Olten, Tel. 06295721 54. felix. wettstein[at]fhnw.ch

werden, dass deutlich weniger häufig Notfallstationen von Kinderkliniken konsultiert werden, wenn die Kinder an Vorsorgeprogrammen teilnehmen.

\section{Elterntrainings als Pflicht?}

Es müssen tatsächlich vermehrte Anstrengungen unternommen werden, damit alle Kinder unter bestmöglichen Bedingungen aufwachsen und sich entwickeln können. Zwangsmassnahmen sind allerdings nicht die geeigneten Mittel dazu, weil sie die Autonomie der Familien einschränken und das Vertrauen zu den Familien beeinträchtigen können. Zudem sind aufwendige Kontrollmechanismen nötig. Für eine effiziente Beratung und Unterstützung von Familien braucht es eine vertrauensvolle Beziehung der Eltern zu den Fachleuten. Dazu ist Freiwilligkeit unerlässlich.

Damit Familien tatsächlich an gesundheitsfördernden und präventiven Programmen teilnehmen, braucht es einerseits gut organisierte interdisziplinäre Strukturen und andererseits gewisse Anreize wie beispielsweise finanzielle Zuwendungen, aber besonders «eine Kultur der Anerkennung statt einer Logik des Verdachts», wie es Hildenbrand genannt hat.

\section{Gehört das Thema in die NCD-Strategie?*}

Das Kosten-Nutzen-Verhältnis der Gesundhetsförderung ist in der frühen Kindheit am höchsten und besonders gross bei Kindern in psychosozialen Risikosituationen. Im Klartext: Pro investierten Franken kommen 8 Franken zurück, bei benachteiligten Kinder sogar 16 Franken. In der Finanzindustrie kann man von solchen Renditen nur träumen. Grund genug, der frühen Kindheit eine zentrale Rolle in der NCD-Strategie zuzuschreiben. Aber strategische Ziele reichen nicht aus, Handeln ist angesagt. 